\title{
Development of a Biosensor for Copper Detection in Aqueous Solutions Using an Anemonia sulcata Recombinant GFP
}

\author{
Tiziana Masullo • Riccardo Puccio • \\ Mariarosaria Di Pierro • Marcello Tagliavia • \\ Paolo Censi • Valeria Vetri • Valeria Militello • \\ Angela Cuttitta $•$ Paolo Colombo
}

Received: 14 October 2013 / Accepted: 1 December 2013 /

Published online: 15 December 2013

(C) Springer Science+Business Media New York 2013

\begin{abstract}
Fluorescent proteins from marine organisms represent potential candidates for biosensor development. In this paper, we described the isolation of a native green fluorescent protein from Anemonia sulcata and the cloning and purification of its equivalent as a recombinant protein in Escherichia coli. Furthermore, the spectroscopic behaviours of the native and recombinant GFPs were investigated as a function of $\mathrm{Cu}^{2+}, \mathrm{Cd}^{2+}, \mathrm{Pb}^{2+}$ and $\mathrm{Ni}^{2+}$ concentration. Our results suggest the high selectivity of both proteins at copper than the other metals and, for the recombinant protein, a great sensitivity at a very low concentration $(0.1-$ $1 \mu \mathrm{M})$. Moreover, starting from these data, using the combination of molecular biology
\end{abstract}

T. Masullo $\cdot$ P. Censi $\cdot$ A. Cuttitta

Istituto per l'Ambiente Marino Costiero, UOS Capo Granitola - Consiglio Nazionale delle Ricerche,

Trapani, Italy

R. Puccio $\cdot$ M. Tagliavia

BioNat Italia S.r.l., Palermo, Italy

M. Di Pierro

Biodiagene S.r.l., Palermo, Italy

P. Censi

Dipartimento di Scienze della Terra e del Mare DiSTeM, Università di Palermo, Palermo, Italy

V. Vetri • V. Militello

Dipartimento di Fisica e Chimica, Università di Palermo, Palermo, Italy

V. Vetri $\cdot$ V. Militello

Istituto di Biofisica-Consiglio Nazionale delle Ricerche, Palermo, Italy

P. Colombo $(\bowtie)$

Istituto di Biomedicina e di Immunologia Molecolare - Consiglio Nazionale delle Ricerche, Via Ugo La Malfa 153, 90146 Palermo, Italy

e-mail: colombo@ibim.cnr.it 
techniques and optical setup, we developed a device for the detection of $\mathrm{Cu}^{2+}$ in water solutions. The quenching effect detected with the device showed that the relative attenuation of the signal $(0.46 \pm 0.02 \mathrm{AU})$ was slightly larger than the data measured by fluorescence spectra $(0.65 \pm 0.03 \mathrm{AU})$. The good sensitivity in the span of two orders of the magnitude of $\mathrm{Cu}^{2+}$ concentration, the fact that the instrument is made up of low-cost and sturdy parts and the selective quenching of rAsGFP to copper ions make this setup suited as a low cost, on-thefield, copper ion-specific biosensor.

Keywords Green fluorescent protein - Recombinant protein - Divalent metal ions · Copper · Quenching · Detector

\section{Introduction}

Heavy and transition metal ions are distributed in biological systems and in the environment, where they can play important roles [1] and can be toxic for several organisms if present at high concentrations. Accurate analytical methods have been developed to detect metal ions in different matrixes or samples [2-4], but they require complex equipments and sophisticated lab manipulations. So, the development of low-cost, user-friendly, quantitative and qualitative detection systems for metal revealing is of paramount importance.

Recently, molecular fluorescence has attracted a great deal of interest in the field of environmental monitoring, and it can be used for the analysis of very low trace elements. For instance, fluorescent proteins (FPs) from different marine organisms may become potential candidates for sensor development. In fact, FPs, such as red and green (GFP) proteins, were used to evaluate the effects of heavy and essential metals on fluorescence intensity [5-12].

Fluorescence quenching has been observed by $\mathrm{Hg}^{2+}[5]$ and $\mathrm{Cu}^{2+}$ on an engineered chimeric GFP harbouring hexahistidine [8]. Moreover, the introduction of metalbinding sites onto the GFP surface places metals in close proximity to chromophore resulted in fluorescence quenching probably induced by energy transfer, at low concentrations (micromolar) of copper ions [10]. Additional mutations on the histidines at different positions have been performed resulting in increased $\mathrm{Cu}^{2+}$-based quenching [12, 13]. Moreover, a dramatic absorbance and fluorescence change upon mercuration in mutant Aequorea victoria GFP was recorded [6]. For the Discosoma striata red fluorescent protein (DsRed) and its variant (DsRed Express, DsRed Monomer, drFP583), fluorescence quenching by $\mathrm{Cu}^{+}$and $\mathrm{Cu}^{2+}$ has been described and the derived copper sensor was 40 times more sensitive than the engineered GFPs [7, 11, 14]. Recently, an Anemonia sulcata GFP has been characterized showing a $50 \%$ of identity to other fluorescent proteins that have been studied for the quenching effect caused by metal ions.

In this report, we describe the development of an optical sensor for copper, using the recombinant $A$. sulcata GFP as a detector. Initially, we isolated the wild-type protein (wtAsGFP) from the organisms, and then, we studied its spectroscopic behaviour by means of the UV-visible spectroscopy in the presence of divalent metal ions. The observed quenching effect induced by copper on the wild-type protein led us to clone, express and produce a recombinant $A$. sulcata GFP ( $r$ AsGFP). Then, we investigated its behaviour in the presence of metal ions, and after evaluating the selectively and sensitivity for copper ions, we set up an optical sensor. 


\section{Materials and Methods}

\section{Protein Extraction and Purification Assay}

Several specimens of $A$. sulcata were collected in the Sicilian Channel. Then, the anemones were promptly transferred to aerated tanks with natural seawater in the laboratory, and the tentacles were cut at the bases. The tentacles were homogenized in Triton X-100, 1× phosphate-buffered saline and a protease inhibitor cocktail (Sigma-Aldrich, Milan, Italy). After centrifugation, the soluble fraction was analysed by means of $10 \%$ sodium dodecyl sulfate polyacrylamide gel electrophoresis (SDS-PAGE) in both reducing and non-reducing conditions. The Versa-Doc Imaging System (Bio-Rad, Milan, Italy) using an FITC fluorophore with a 530BP blue LED was used to detect the fluorescent protein. The raw extract was partially purified using a gel filtration chromatography. Sephadex G75 (3,000-80,000 Da) (GE Healthcare, Hertfordshire, UK) was used in the stationary phase, whereas $20 \mathrm{mM} 4$ morpholineethanesulfonic acid hydrate (MES) (AppliChem, Milan, Italy) pH 7 was used in the eluent phase. A total of $12.2 \mathrm{mg}$ of raw extract $[17.2 \mathrm{mg} / \mathrm{mL}]$ was injected into a column of $16 \times 1.2 \mathrm{~cm}$, with a flow rate set of $0.5 \mathrm{~mL} / \mathrm{min}$ and the fraction collector at $1 \mathrm{~mL}$ of volume solution.

\section{Isolation, Cloning and Purification}

Total RNA was extracted from tentacle tissue using a TRIzol reagent, according to the manufacturer's instructions (Invitrogen, Milan, Italy). Subsequently, it was reverse transcribed using the SuperScript ${ }^{\mathrm{TM}}$ II Reverse Transcriptase and Oligo-d(T) Kit (Invitrogen, Milan, Italy). The Anemonia cDNA library was screened for the asFP499 coding region (GenBank: AAN52735.1) using Pure Taq Ready-To-Go PCR beads (GE Healthcare Life Sciences, UK) and specific forward (5'-cgcGGATCCATGTATCCTTCCATCAAG-3') and reverse (3'gcgGGATCCTCAGTTATGTCCTAATTTCG-5') primers (underlined letters indicate the BamH 1 restriction enzyme sites introduced for cloning in the expression vector; lowercase letters indicate the nucleotides introduced to improve the restriction enzyme's recognition; and the start and stop codons are indicated in bold). The coding sequence for AsGFP was ligated in a pJET1.2/Blunt vector using the CloneJet PCR Cloning Kit (Fermentas, Milan, Italy) and transformed into a DH5 $\alpha$ Escherichia coli strain (Invitrogen, Milan, Italy). Then, the coding reading frame of the recombinant clones was checked by sequencing. The recombinant plasmid was purified and digested with the BamH1 restriction enzyme, and the cDNA was cloned in the same restriction site in the pQE30 expression vector (Qiagen, Milan, Italy). The recombinant AsGFP was expressed as a His-tagged fusion protein using the M15 E. coli strain (Qiagen, Milan, Italy). Production of the rAsGFP was performed according to the protocol suggested by Peternel and coworkers [15]. Briefly, the inoculum was incubated for $16 \mathrm{~h}$ at $25^{\circ} \mathrm{C}$. Then, the bacterial pellet was re-suspended in $1 \times$ phosphate buffer $\mathrm{pH} 7.4,0.5 \mathrm{M} \mathrm{NaCl}$ and $10 \mathrm{mM}$ imidazole, and the cells were disrupted (six cycles of $30 \mathrm{~s}$ at $70 \%$ amplitude, with a 30-s pause between the cycles) using a sonicator device (Bandelin Sonoplus Ultrasonic homogenizer HD 2070, Berlin, Germany). Cell debris was removed by centrifugation at $10,000 \mathrm{rpm}$ for $20 \mathrm{~min}$ at $4{ }^{\circ} \mathrm{C}$. After centrifugation, the soluble fraction was filtered through a sterile 5- $\mu \mathrm{m}$ syringe disc-type filter (Corning Life Sciences, Lowell, MA) and used for subsequent affinity purification of the protein via nickel affinity chromatography (GE Healthcare, Hertfordshire, UK) using the N-terminal His-tag of the protein. Purification was performed by applying a discontinuous imidazole gradient $(50-500 \mathrm{mM})$ following the manufacturer's instructions. Fractions were checked on a $10 \%$ SDS-PAGE, and aliquots 
containing the rAsGFP were loaded onto a G25 desalting column (GE Healthcare, Hertfordshire, UK) to allow buffer exchange (20 mM MES pH 7). The purity and molecular weight of the protein were confirmed by SDS-PAGE followed by Coomassie brilliant blue staining and Bradford assay.

\section{Spectroscopy}

Absorption and fluorescence measurements were carried out on partially purified wild-type (wtAsGFP) and recombinant (rAsGFP) proteins. Absorption spectroscopy was performed on a UV-Vis spectrophotometer (Shimadzu) in a range from 190 to $900 \mathrm{~nm}$. Fluorescence measurements were performed on a Cary Eclipse Fluorescence Spectrofluorometer (Varian) $\left(B W_{\lambda \mathrm{ex}}=5 \mathrm{~nm}, \mathrm{BW}_{\lambda \mathrm{em}}=5 \mathrm{~nm}\right)$ using a cuvette with a $1-\mathrm{cm}$ path length. All the experiments were performed in three replicates. The experimental errors were evaluated as 5 and $3 \%$ of intensity value for fluorescence and scattering data, respectively. It should be noted that fluorescence spectra were not corrected for instrumental response because only variations in fluorescence intensity were taken in account. The suitable aliquot $\mathrm{Cd}\left(\mathrm{NO}_{3}\right)_{2}, \mathrm{~Pb}\left(\mathrm{NO}_{3}\right)_{2}$, $\mathrm{Ni}\left(\mathrm{NO}_{3}\right)_{2}$ or $\mathrm{CuCl}_{2}$ (Merck, Germany) solutions were added to $1.5 \mu \mathrm{M}$ wtAsGFP and $r$ AsGFP in $20 \mathrm{mM}$ MES pH 7.0 samples (rt) in order to vary the metal concentration in the range of $0.1-50 \mu \mathrm{M}$. Metal solutions were mixed with samples right before measurements. It was verified that metal solution at the observed concentration had no remarkable fluorescence signal. Supplementary control experiments were performed with $\mathrm{HNO}_{3}$ and $\mathrm{NaCl}$ (concentration range from 0.3 to $1,000 \mu \mathrm{M}$ ). In some circumstances, ethylenediaminetetraacetic acid (EDTA) (AppliChem, Milan, Italy) was added to the sample at a final concentration of $0.5 \mathrm{mM}$ as a further control experiment.

Within the experimental error, all fluorescence experiments were performed at constant protein concentration. In each experiment, fluorescence quenching is calculated by using the maximum intensity value of the fluorescence emission band, in the absence of metal ions, as a reference.

\section{Data Processing and Stern-Volmer Plot}

Stern-Volmer plots were generated for $1.5 \mu \mathrm{M}$ of $w t$ AsGFP and $r$ AsGFP in the presence of $\mathrm{Cu}^{2+}, \mathrm{Pb}^{2+}, \mathrm{Cd}^{2+}$ and $\mathrm{Ni}^{2}$ (range from 0.1 to $50 \mu \mathrm{M}$ ), using Eq. (1), in which $F_{0}$ is the measured fluorescence intensity without quencher present, $F$ is the measured fluorescence intensity in presence of quencher, $[Q]$ is the quencher concentration and $K_{\mathrm{sv}}$ is the SternVolmer constant. After plotting $F_{0} / F$ against [ $Q$ ], the slope was determined to give the value of $K_{\mathrm{sv}}$ that is indicative of the quencher affinity to the proteins.

The dissociation equation for copper $\left(K_{\mathrm{d}}\right)$ was determined by fitting the fluorescence quenching data to the following Eq. (2) [16], in which $\Delta F_{\mathrm{a}}$ is the change in fluorescence amplitude, $F_{\mathrm{i}}$ the initial fluorescence without any copper, $[\mathrm{Cu}]$ is the maximum copper concentration and $F$ is the observed fluorescence.

$$
\begin{aligned}
& \frac{F_{0}}{F}=1+K_{\mathrm{sv}}[Q] \\
& F=F_{\mathrm{i}}-\frac{\Delta F_{\mathrm{a}}}{\frac{K_{\mathrm{d}}}{[\mathrm{Cu}]}-1}
\end{aligned}
$$


Biosensor Development and Measurement Procedure

The instrument was set up with the following components: a LED source (PW about $470 \mathrm{~nm}$ ), a photodiode with a proprietary amplification circuit and two optical blocks placed in a $45^{\circ}$ geometry. The instrument is designed to focus the excitation light beam from the source on a $50-\mu \mathrm{L}$ drop sample sitting on a microscope slide, to collect the fluorescence emitted from the sample and to focus it on a photodiode. The light from the LED was collimated by a planoconvex lens, filtered by a bandpass filter (center wavelength $(\mathrm{CWL})=470 \mathrm{~nm}$, full width at half maximum $(\mathrm{FWHM})=10 \mathrm{~nm})$ and focused on the sample by a plano-convex lens. The fluorescence emission from the sample was collimated by a plano-convex lens, filtered by a bandpass filter $(\mathrm{CWL}=508 \mathrm{~nm}, \mathrm{FWHM}=10 \mathrm{~nm})$ and focused on the photodiode by a planoconvex lens. The resulting current from the photodiode was amplified and a transimpedance amplifier converted it into a voltage signal, which was then measured by a simple multimeter. Figure 5a shows both a diagram of the working principles of the instrument and a picture of the realized prototype (Fig. 5b).

Analyses were performed using a solution containing $1.5 \mu \mathrm{M}$ rAsGFP in $20 \mathrm{mM}$ MES pH 7. The effect of $\mathrm{CuCl}_{2}$ was tested with concentrations ranging from 0.1 to $10 \mu \mathrm{M}$. Drops $(50 \mu \mathrm{L})$ of each solution were deposited on microscope slide wells, and the slide was placed on the focus spot of the incident light from the LED. For each solution at a specific $\mathrm{CuCl}_{2}$ concentration, a voltage reading resulting from the fluorescence of the sample was taken. Each voltage value was then normalized to the voltage reading from a sample without copper. Four different sets of measurements were taken to verify the repeatability of the instrument; for each normalized voltage measured at a certain $\mathrm{CuCl}_{2}$ concentration, the four readings were averaged, and the standard deviation between them was taken as an estimate of the measurement error.

\section{Results}

\section{Biochemical Characterization of Wild-Type AsGFP}

As a first approach, we isolated total proteins from the $A$. sulcata tentacles. By means of gel filtration chromatography, the raw extract was fractioned allowing the collection of several fractions, but only two of them (about of $2 \mathrm{mg}$ of proteins) showed fluorescence. These two samples were pooled and used as partially purified native protein. SDS-PAGE of a raw extract and partially purified native protein (wtAsGFP) showed a single fluorescent band migrating as tetramer, with an apparent molecular weight (MW) of $\sim 124 \mathrm{kDa}$ (Fig. 1a).

\section{Spectroscopic Analysis and Data Processing on the Wild-Type AsGFP}

Emission spectrum of the $w t$ AsGFP showed two peaks at 500 and $520 \mathrm{~nm}$, respectively $\left(\lambda_{\mathrm{ex}}=\right.$ $460 \mathrm{~nm}$ ) (Fig. 1b). The ratio between the two peaks changes as a function of excitation wavelength (Fig. 1b, inset). We assured the stability of the wtAsGFP in $20 \mathrm{mM} \mathrm{MES} \mathrm{buffer} \mathrm{pH}$ 7 checking that the emission spectrum did not present any detectable variation as a function of time up to $24 \mathrm{~h}$ (data not shown). In all experiments, no evident changes in the shape and position of fluorescence emission peaks were observed. In particular, changes in the wtAsGFP fluorescence spectrum were shown as a function of copper concentration in the range of 2$30 \mu \mathrm{M}$ (Fig. 1c). It can be observed that after incubation with the copper ions, the emission spectrum of wtAsGFP did not change shape and position but a decrease in intensity is clearly 


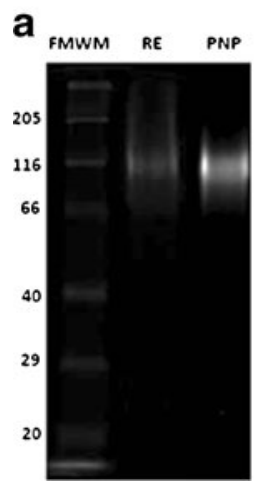

b

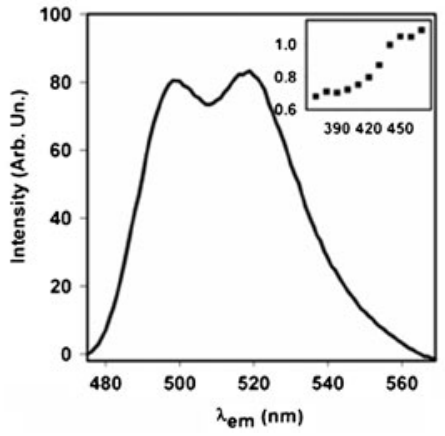

C

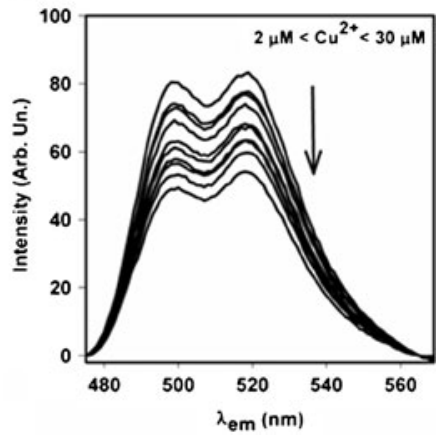

Fig. 1 Characterization of native protein: a SDS-PAGE in no reducing conditions. Line 1: Fluorescent molecular weight marker (20-205 kDa); Line 2: raw extract $(R E)$; Line 3: partially native purified protein $(P N P)$; b Emission spectrum of $w t$ AsGFP $\left(\lambda_{\mathrm{ex}}=460 \mathrm{~nm}\right)$. The inset shows details on the normalized intensity of peaks ratio changes as a function of excitation wavelength; c Emission spectrum of $w t$ AsGFP ( $\left.\lambda_{\mathrm{ex}}=460 \mathrm{~nm}\right)$ in the presence of copper. The arrow shows the quenching of fluorescence as a function of gradual copper ion concentration increase from 2 to $30 \mu \mathrm{M}$

demonstrated. The emission intensity was restored to its initial value by adding EDTA as a sequestering agent to the sample (data not shown). A more detailed analysis of the fluorescence quenching as a function of copper concentration for wild-type protein is shown in Fig. 2a. A decrease in fluorescence intensity of $24 \%(0.76 \pm 0.04 \mathrm{AU})$ was obtained for $10 \mu \mathrm{M}$ of copper (1.5:10 wt protein/metal ratio), whereas a quenching of $35 \%$ was recorded after $30 \mu \mathrm{M}$ copper ion addition (1.5:30 wt protein/metal ratio) (data not shown). In the same experimental conditions, the fluorescence reduction caused by lead, cadmium and nickel $(35 \mu \mathrm{M})$ was definitively less than that measured in the presence of copper. The quenching trend was the same for all tested metals; moreover, the fluorescence intensity did not show a significant decrease (about $17 \%$ for $\mathrm{Pb}^{2+}$ and $8 \%$ for $\mathrm{Cd}^{2+}$ and $\mathrm{Ni}^{2}$ ) (data not shown). SternVolmer plots confirmed these results. They were generated by measuring the fluorescence of $w t$ AsGFP upon addition of all metals at the same temperature (Fig. 2b). Linear plots were obtained, indicating that only one type of quenching occurred. The higher $K_{\mathrm{sv}}$ constants were obtained for copper ion (Table 1).

a

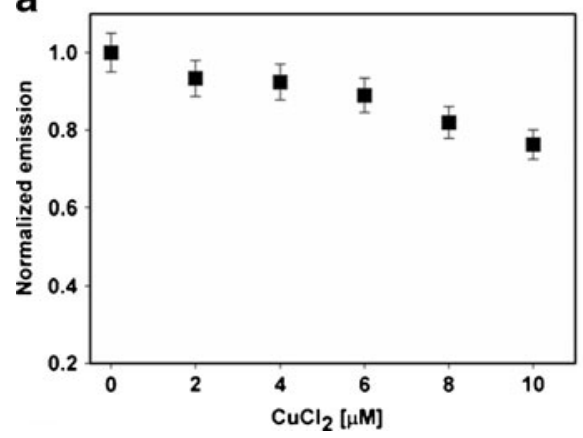

b

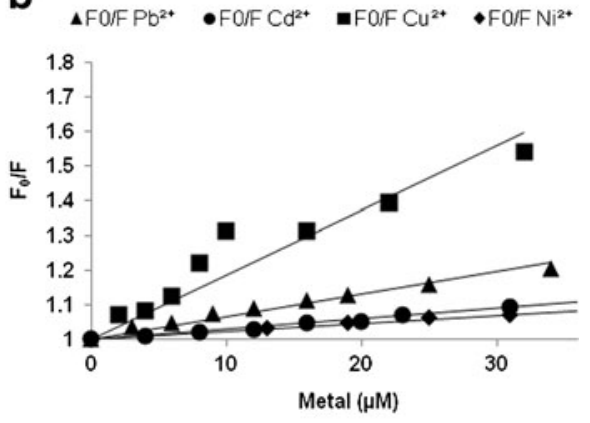

Fig. 2 a $w t$ AsGFP fluorescence quenching as a function of copper concentration (from 0.1 to $10 \mu \mathrm{M}$ ). b SternVolmer plots of wtAsGFP as a function of $\mathrm{Cu}^{2+}, \mathrm{Pb}^{2+}, \mathrm{Cd}^{2+}$ and $\mathrm{Ni}^{2+}$ concentration range from 1 to $35 \mu \mathrm{M}$ 
Table 1 Stern-Volmer constants $\left(K_{\mathrm{sv}}\right)$ of $\mathrm{Cu}^{2+}, \mathrm{Pb}^{2+}, \mathrm{Cd}^{2+}$ and $\mathrm{Ni}^{2+}$ for the $w t$ AsGFP and linear regression correlation coefficient $\left(R^{2}\right)$

\begin{tabular}{lll}
\hline Metal & $K_{\mathrm{sv}}\left(\mathrm{M}^{-1}\right)$ & $R^{2}$ \\
\hline $\mathrm{Cu}^{2+}$ & $2 \times 10^{4}$ & 0.86 \\
$\mathrm{~Pb}^{2+}$ & $0.6 \times 10^{4}$ & 0.97 \\
$\mathrm{Cd}^{2+}$ & $0.3 \times 10^{4}$ & 0.99 \\
$\mathrm{Ni}^{2+}$ & $0.2 \times 10^{4}$ & 0.95 \\
\hline
\end{tabular}

\section{Molecular Characterization and Expression of a Recombinant AsGFP}

A cDNA of 687 bp was isolated from total RNA isolated from $A$. sulcata tentacles containing an ORF of 228 aa giving a protein with a putative MW of $24 \mathrm{kDa}$. Sequence analysis performed with the BLAST algorithm showed that our AsGFP showed a certain degree of homology to several other GFPs. In particular, a $99 \%$ identity to the asFP499 protein (GenBank: AAG412051) was shown with a single amino acid change (Thr-108 to Ala-108) outside the chromophore (Fig. 3a). Table 2 displays the percentage of identity and homology to other GFPs known for their selectivity to copper ions. The identity ranged from 73 to $48 \%$.

The rAsGFP was expressed as a His-tagged protein in E. coli at a good yield allowing the production of about $0.5 \mathrm{mg} / \mathrm{L}$ of highly purified protein. After purification, a single band of about $24 \mathrm{kDa}$ was observed on SDS-PAGE and Coomassie brilliant blue staining (Fig. 3b).

Fluorescence Measurements on the Recombinant AsGFP

$r$ AsGFP presents an intense fluorescence emission band, once excited in the visible range. In Fig. 3c, we report the fluorescence emission band (range from 470 to $580 \mathrm{~nm}$ ) of $1.5 \mu \mathrm{M}$ $r$ AsGFP in $20 \mathrm{mM}$ MES buffer $\mathrm{pH} 7$ excited at $\lambda_{\mathrm{ex}}=460$. As can be seen, the emission spectrum is in the same region as the one relative to the wtAsGFP, but it presents a single main peak at about $500 \mathrm{~nm}$. In Fig. 3d, we show the fluorescence spectrum of rAsGFP at an increasing concentration of $\mathrm{Cu}^{2+}$ ion in solution. As observed for $w t$ AsGFP, the shape of the emission signal and its position do not change as a function of metal concentration, but, again, a significant decrease in fluorescence intensity is monitored as a function of metal concentration (from 0.1 to $10 \mu \mathrm{M}$ ). The addition of EDTA to the sample brings back fluorescence intensity to its initial value, demonstrating that the quenching effect was due to the presence of the metal ion (data not shown). The spectroscopic analysis was performed on recombinant protein in the same experimental conditions used for the wild-type protein. Data show a similar behaviour with a high quenching effect at lower copper concentrations compared to those used for wild-type protein (Fig. 4a). Furthermore, as shown in the inset, a linear dependence of fluorescence quenching was observed in the range $0-1 \mu \mathrm{M}$ of copper. In particular, a $35 \%$ of quenching $(0.65 \pm 0.03 \mathrm{AU})$ was observed at $1 \mu \mathrm{M}$ of copper while, at higher concentration, the fluorescence signal keeps in decreasing until a $65 \%(0.35 \pm 0.02 \mathrm{AU})$ at $10 \mu \mathrm{M}$ of copper.

Stern-Volmer plot was generated for $r$ AsGFP, and the linear plot was compared with the $w t$ AsGFP fit (Fig. 4b). The $K_{\mathrm{sv}}$ values were $1.9 \times 10^{5} \mathrm{M}^{-1}$ for $r$ AsGFP and $3 \times 10^{4} \mathrm{M}^{-1}$ for the $w t$ AsGFP. The dissociation constant of copper binding site on the $r$ AsGFP was calculated as $15 \pm 0.7 \mu \mathrm{M}$.

\section{Optical Detector and Measurement Results}

According to our previously reported data showing that both native and recombinant AsGFP display a peculiar sensitivity to $\mathrm{Cu}^{2+}$ and that the recombinant one shows an increased 
a

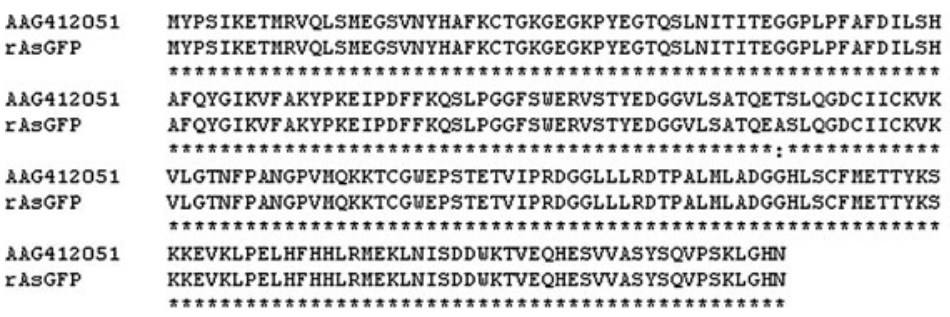

b

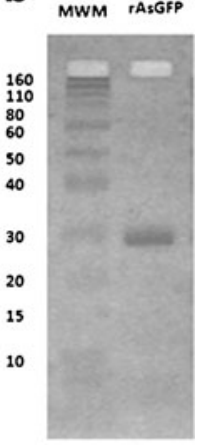

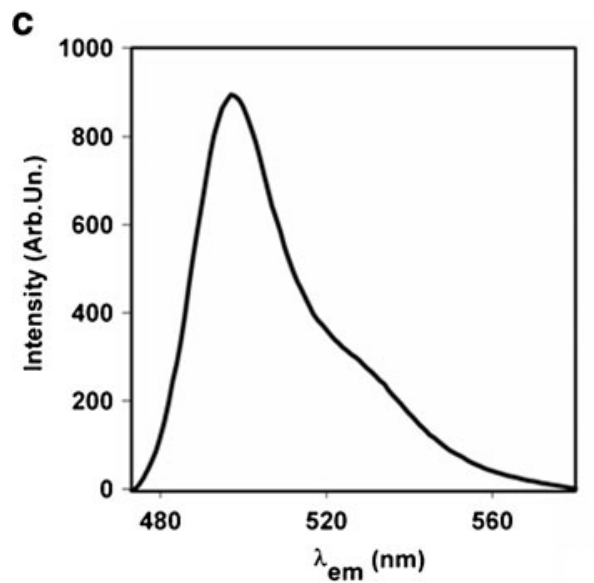

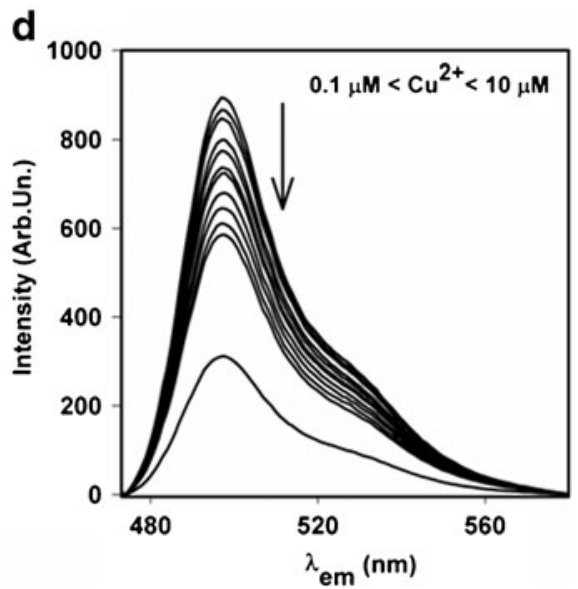

Fig. 3 Characterization of recombinant protein: a Alignment sequence (ClustalW) between asFP499 (GenBank: AAG412051) and recombinant purified protein; b SDS-PAGE in reducing conditions. Line 1: Molecular weight marker (3.5-260 kDa); Line 2: recombinant purified green fluorescent protein $(r A s G F P)$; c Emission spectrum of $r$ AsGFP $\left(\lambda_{\mathrm{ex}}=460 \mathrm{~nm}\right)$; d Emission spectrum of $r \operatorname{AsGFP}\left(\lambda_{\mathrm{ex}}=460 \mathrm{~nm}\right)$ in the presence of copper. The arrow shows the fluorescence quenching as a function of gradual copper ion concentration increase from 0.1 to $10 \mu \mathrm{M}$

sensitivity to the metal when compared to the w.t. AsGFP, we decided to test the possibility to design an optical sensor for the detection of copper ions in an aqueous environment using the

Table 2 Comparison of identity and homology percentage between the rAsGFP and other fluorescent proteins

\begin{tabular}{lll}
\hline Accession number & Identity, \% & Homology, \% \\
\hline AAG41205.1 & 100 & 100 \\
rAsGFP & 99 & 99 \\
AAO16871.1 & 73 & 83 \\
AAA27722.1 & 60 & 70 \\
AAG16224.1 & 59 & 69 \\
ACF35425.1 & 53 & 70 \\
Q9U6Y8.1 & 52 & 70 \\
AAF62891.1 & 50 & 70 \\
ACU30027.1 & 48 & 65
\end{tabular}



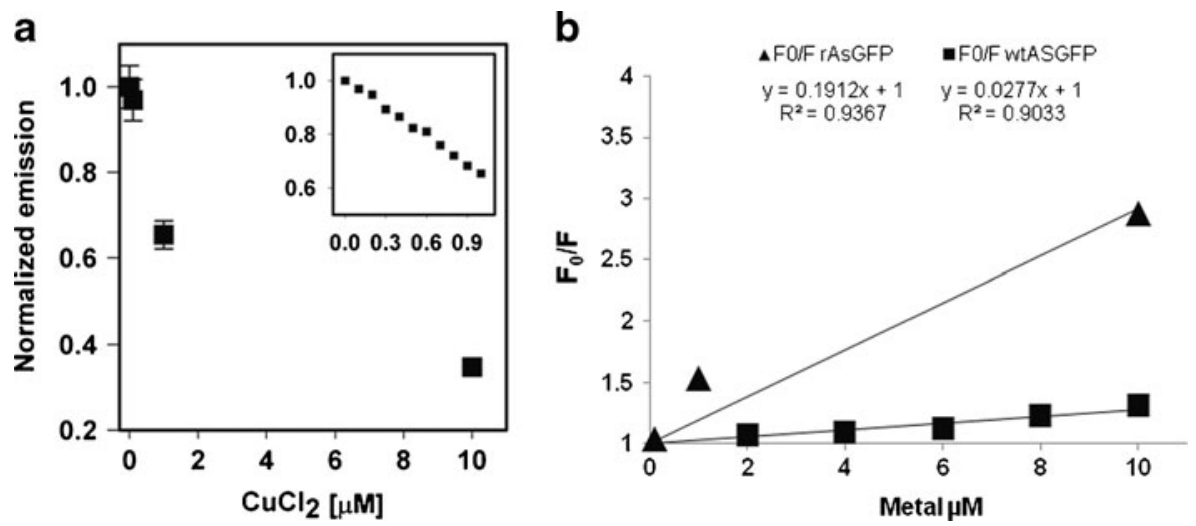

Fig. 4 a $r$ AsGFP fluorescence quenching as a function of copper concentration (range $0.1-10 \mu \mathrm{M}$ ). The inset shows details on the emission changes at very low copper concentration from 0.1 to $1 \mu \mathrm{M}$. b Stern-Volmer plots of $r$ AsGFP as a function of $\mathrm{Cu}^{2+}$ against $w$ tAsGFP

rAsGFP. As schematically depicted in Fig. 5a, the development of a protein-based copper sensor was carried out by depositing the rAsGFP on the well of a microscope slide and exposing the focus spot of the incident light of a LED $(470 \mathrm{~nm})$. The relative voltage reading resulting from the fluorescence of the recombinant protein against $\mathrm{Cu}^{2+}$ concentration was examined. The average of the four normalized voltage readings measured at various $\mathrm{Cu}^{2+}$ concentrations and their standard deviations are shown in Fig. 6. In line with what was observed with standard fluorescence measurements, there was a good linearity in the $0.1-$ $1 \mu \mathrm{M}$ range (see inset in Fig. 6) and good sensitivity up to $10 \mu \mathrm{M}$, where a plateau in fluorescence quenching was reached. Compared to the results obtained with fluorescence spectra, the relative attenuation of the signal was slightly larger $(0.46 \pm 0.02 \mathrm{AU}$ compared to $0.65 \pm 0.03 \mathrm{AU}$ at 1.5:1 recombinant protein/metal ratio); this effect could be attributed to a deviation from linearity in the low intensity range of the detector.

\section{Discussion}

Anemonia spp. contain green fluorescence proteins whose primary sequences and spectroscopic features have been already described, but little is known about the quenching of

a

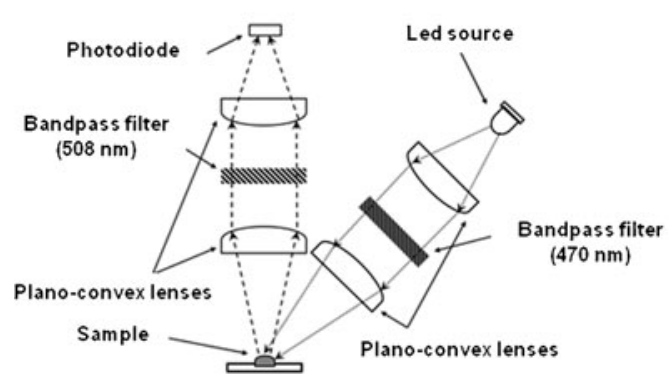

b

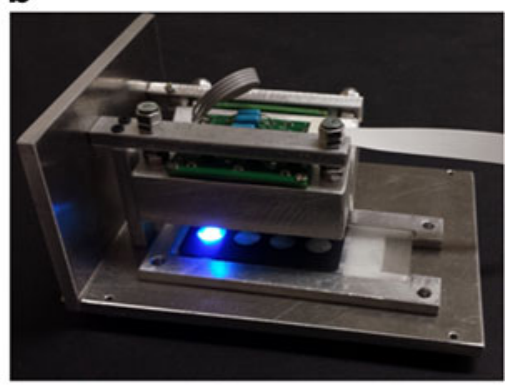

Fig. 5 a Schematic representation of the instrument. b Picture of the prototype 
Fig. 6 rAsGFP fluorescence quenching measured as a function of copper concentration (range $0.1-10 \mu \mathrm{M}$ ) using the engineered detector. The inset shows the fluorescence measured in a very low concentration range of copper from 0.1 to $1 \mu \mathrm{M}$

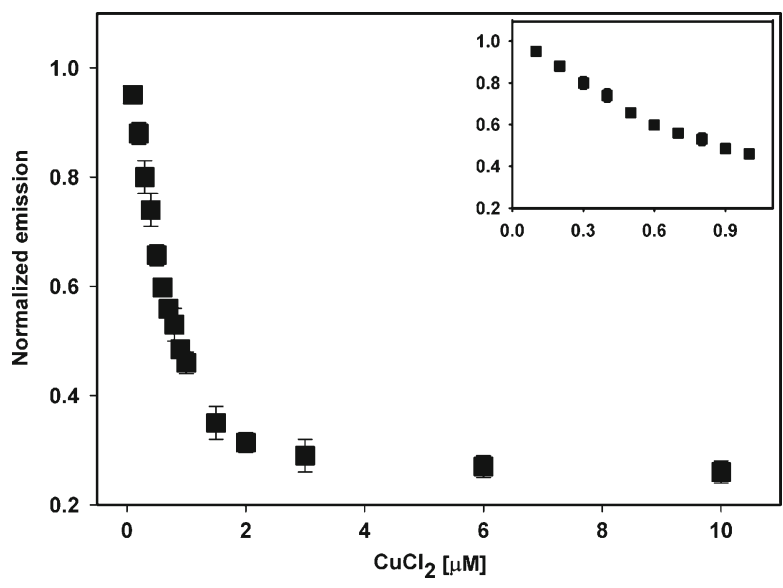

fluorescence induced by metal ions. The aim of our work was to exploit the effect of several metal ions on the fluorescence spectra of an A. sulcata GFP on the attempt to develop an optical sensor for the detection of metal ions.

To address this question, firstly we purified a native AsGFP from A. sulcata tentacles. SDSPAGE analysis demonstrated that the wtAsGFP displays a tetrametric conformation. This datum is consistent to other reports where it has been shown that all known Anthozoa GFP-like proteins form oligomers and exist mostly as tetramers [17-22]. Moreover, by means of DNA recombinant technologies, a cDNA expressing an AsGFP was isolated and its coding sequence expressed in E. coli as a His-tagged protein. Sequence alignment with other FPs showed a range of identity from 73 to $48 \%$. Moreover, our sequence was $99 \%$ homologous to the asFP499 GFP previously identified in A. sulcata with only one amino acid difference (Thr- ${ }^{108}$ to Ala) in a residue that it does not seem to be implicated in any mechanism of autocatalytic chromophore formation [23-25]. The spectral properties of the wild-type and recombinant proteins were studied by means of UV-Vis spectroscopy showing a different shape of spectrum and position of the emission peaks. In fact, using the wtAsGFP, we recorded two emission peaks rather than a single peak as shown for the rAsGFP, at the same excitation wavelength. Wiedenmann and colleagues [21] supposed the existence of two different forms of the same GFP in the tentacle tissues that can be distinguished by their spectral properties, although they could not be biochemically separated. In fact, they assumed the existence of a second stable conformation of asFP499, with red-shifted fluorescence. In line with data reported in Wiedenmann and colleagues [21], we have found that the ratio between the two peaks changes as a function of excitation wavelength (inset in Fig. 1b). Small differences between our data and the spectra reported in Wiedenmann et al. [21] can be attributed to different experimental conditions (e.g. presence of other components, buffer and $\mathrm{pH}$ ) [26].

To study the possible effect of quenching of fluorescence induced by different metal ions on wtand rAsGFP, we recorded the emission spectra for both proteins after the addition of increasing concentration of metals such as $\mathrm{Cd}\left(\mathrm{NO}_{3}\right)_{2}, \mathrm{~Pb}\left(\mathrm{NO}_{3}\right)_{2}, \mathrm{Ni}\left(\mathrm{NO}_{3}\right)_{2}$ or $\mathrm{CuCl}_{2}$. In this set of experiments, we observed a similar trend of fluorescence reduction using lead, cadmium and nickel as quenchers, suggesting that those metals interact with the proteins but with different and probably non-specific effects. These data were in line with findings in other investigated FPs $[6,7,10,12]$.

On the other hand, $\mathrm{Cu}^{2+}$ showed a different behaviour causing a significant reduction of the protein emission intensity. In particular, the wt- and rAsGFP proteins showed a similar $\mathrm{Cu}^{2+}$ induced quenching effect indicating that they can act as copper indicators in different ranges. 
The Stern-Volmer plot confirmed these results (Fig. 4b); in fact the $K_{\mathrm{sv}}$ constant for copper is higher than the one for other metals indicating a better affinity of copper quencher to the protein site than the other metals (Table 1). The calculated dissociation constant for $\mathrm{Cu}^{2+}$ was similar to that obtained for other proteins such as EGFP, DsRed, muted drFP583 and Rmu13 [7, 13], while for DsRed Monomer and DsRed Express, we observed a higher value [9]. Other naturally available copper-binding proteins and peptides have reported $K_{\mathrm{d}}$ values ranging from $10^{-6}$ to $10^{-15} \mathrm{M}$ for proteins and up to $10^{-17} \mathrm{M}$ for peptides [27, 28]. In summary, our results have shown that fluorescence measurements on both $w t$ AsGFP and $r$ AsGFP protein revealed that (a) $\mathrm{Cu}^{2+}$ presence induces a large, concentration-dependent quenching in AsGFP fluorescence; (b) the observed effect due to $\mathrm{Cu}^{2+}$ seems to be somehow specific because it is larger than the one induced by other divalent metal ions that show homologous behaviour. Moreover, the larger fluorescence signal of recombinant protein in the absence of a metal with respect to the wild type and its more sensitivity to copper ions could be explained hypothesising a different conformation in the chromophore(s) or in its/their environment which could provide different interaction sites or mechanisms with copper.

Larger changes in protein fluorescence induced by $\mathrm{Cu}^{2+}$ suggest its more specific role as a donor group with respect to $\mathrm{Pb}^{2+}, \mathrm{Cd}^{2+}$ and $\mathrm{Ni}^{2+}$, explaining their limited quenching effects. Furthermore, a possible structural significance in terms of a " $\mathrm{Cu}^{2+}$-protein" bond with an inner-sphere mechanism is suggested by recognized spectroscopic evidence. It is largely accepted that $\mathrm{Cu}^{2+}$ ions may interact with proteins causing changes in their tertiary and secondary structure, but also favouring the formation of strong intermolecular H-bonds, which can also lead towards gelation [29, 30]. The observed fluorescence changes may be due to local differences in protein conformation or in the metal-protein interaction sites. This may induce changes in the energy of the states involved in electronic transition. For example, different positions in fluorescence spectra were recently attributed to a reversible protonation and cis-trans isomerization of the chromophore in several GFP molecules causing an on/off switching in response to illumination at a different wavelength [31]. Typically, fluorescence quenching can be caused by a variety of molecular interactions, e.g. ground-state complex formation, excited-state reaction, molecular rearrangements, energy transfer and collisional (dynamic) quenching. Structural motifs having affinity for $\mathrm{Cu}^{2+}$ are frequently composed of the side chains of His, Tyr, Glu, Asp, Cys and sometimes Asn and Gln [32, 33]. It is noteworthy to mention that our AsGFP is produced as a His-tag protein, so it can be suggested that the presence of additional histidine residues can enhance the quenching effect as shown for other GFPs. Many authors have confirmed a selective bond of copper with mutated GFP, in which some residues are changed to histidines $[8,10,13]$, and have emphasized how the location and distance of the imidazole ring need to be very close to the GFP fluorophore in order to effectively quench the fluorescence [12].

The concurrence of the selective and enhanced quenching of rAsGFP with respect to copper ions and the possibility to produce large amounts of highly purified protein opened the way for the design of a biosensor for copper quantification in aqueous solutions. The limit of copper concentration above which aqueous solution may pose a threat to human health has been set to $31 \mu \mathrm{M}$ by the World Health Organization [34]. Since the developed biosensor was able to measure the presence of copper in the $0.1-10 \mu \mathrm{M}$ range, it could perform copper quantification in aqueous solutions both at toxic and nontoxic concentrations by opportune dilution of the sample. The developed set up is (a) compact (the footprint of the prototype, shown in Fig. 5 b, is about $12.5 \times 9 \mathrm{~cm}$ ), (b) has a measuring range of up to two orders of magnitude of copper concentration and (c) is made up of low-cost and sturdy parts, such as fixed lenses and filters and a simple photodiode as a light detector. Moreover, the whole setup can be handled by a simple microcontroller interface, and it only takes a few seconds to 
measure the relative quenching of the sample. It could therefore be suited as a low-cost, on-thefield, copper ion-specific biosensor. It could also be used as a metal ion biosensor coupled with any other fluorescent protein that has metal-specific fluorescence quenching by simply replacing the excitation/emission filters, allowing for quick comprehensive testing of aqueous solutions for a set of specific metals.

\section{Conclusions}

In this paper, we reported the development of a biosensor based on the combination of molecular biology techniques and optical setup. The integration of different techniques into a single system led to the detection of $\mathrm{Cu}^{2+}$ ions in water solutions with a range of sensitivity similar to other bench-top, complex and expensive instruments. The simplicity of the instrument design will enable further miniaturization and the reduction of costs, with the aim of producing a device that can be used for diagnosis in a variety of settings.

Acknowledgments We thank Dr. Salvatore Mazzola for critical reading of the manuscript and Dr. Aldo Nicosia and Dr. Monica Salamone for helpful discussion. This work was supported by PO FERS 2007/2013 Linea di intervento 4.1.1.2.

\section{References}

1. Trautwein, A. X. (1997). Bioinorganic chemistry: transition metals in biology and their coordination chemistry. Wiley-VCH, Weinheim.

2. Becker, J. S., Zoriy, M. V., Pickhardt, C., Palomero-Gallagher, N., \& Zilles, K. (2005). Imaging of copper, zinc, and other elements in thin section of human brain samples (hippocampus) by laser ablation inductively coupled plasma mass spectrometry. Analytical chemistry, 77, 3208-3216.

3. Rao, G. P., Seshaiah, K., Rao, Y. K., \& Wang, M. C. (2006). Solid phase extraction of Cd, Cu, and Ni from leafy vegetables and plant leaves using amberlite XAD-2 functionalized with 2-hydroxy-acetophenonethiosemicarbazone (HAPTSC) and determination by inductively coupled plasma atomic emission spectroscopy. Journal of agricultural and food chemistry, 54, 2868-2872.

4. Xiang, Y., Li, Z., Chen, X., \& Tong, A. (2008). Highly sensitive and selective optical chemosensor for determination of $\mathrm{Cu} 2+$ in aqueous solution. Talanta, 74, 1148-1153.

5. Bozkurt, S. S., \& Cavas, L. (2009). Can Hg(II) be determined via quenching of the emission of green fluorescent protein from Anemonia sulcata var. smaragdina? Applied biochemistry and biotechnology, 158, 51-58.

6. Chapleau, R. R., Blomberg, R., Ford, P. C., \& Sagermann, M. (2008). Design of a highly specific and noninvasive biosensor suitable for real-time in vivo imaging of mercury(II) uptake. Protein science: a publication of the Protein Society, 17, 614-622.

7. Eli, P., \& Chakrabartty, A. (2006). Variants of DsRed fluorescent protein: development of a copper sensor. Protein science: a publication of the Protein Society, 15, 2442-2447.

8. Isarankura-Na-Ayudhya, C., Tantimongcolwat, T., Galla, H. J., \& Prachayasittikul, V. (2010). Fluorescent protein-based optical biosensor for copper ion quantitation. Biological trace element research, 134, 352-363.

9. Rahimi, Y., Goulding, A., Shrestha, S., Mirpuri, S., \& Deo, S. K. (2008). Mechanism of copper induced fluorescence quenching of red fluorescent protein, DsRed. Biochemical and biophysical research communications, 370, 57-61.

10. Richmond, T. A., Takahashi, T. T., Shimkhada, R., \& Bernsdorf, J. (2000). Engineered metal binding sites on green fluorescence protein. Biochemical and biophysical research communications, 268, 462-465.

11. Sumner, J. P., Westerberg, N. M., Stoddard, A. K., Hurst, T. K., Cramer, M., Thompson, R. B., et al. (2006). DsRed as a highly sensitive, selective, and reversible fluorescence-based biosensor for both $\mathrm{Cu}(+)$ and $\mathrm{Cu}(2+)$ ions. Biosensors \& bioelectronics, 21, 1302-1308.

12. Tansila, N., Becker, K., Isarankura Na-Ayudhya, C., Prachayasittikul, V., \& Bulow, L. (2008). Metal ion accessibility of histidine-modified superfolder green fluorescent protein expressed in Escherichia coli. Biotechnology letters, 30, 1391-1396. 
13. Balint, E. E., Petres, J., Szabo, M., Orban, C. K., Szilagyi, L., \& Abraham, B. (2013). Fluorescence of a histidine-modified enhanced green fluorescent protein (EGFP) effectively quenched by copper(II) ions. Journal of fluorescence, 23, 273-281.

14. Fradkov, A. F., Verkhusha, V. V., Staroverov, D. B., Bulina, M. E., Yanushevich, Y. G., Martynov, V. I., et al. (2002). Far-red fluorescent tag for protein labelling. The Biochemical journal, 368, 17-21.

15. Peternel, S., Gaberc-Porekar, V., \& Komel, R. (2009). Bacterial growth conditions affect quality of GFP expressed inside inclusion bodies. Acta Chimica Slovenica, 56, 860-867.

16. Fersht, A. (1999). Structure and mechanism in protein science: a guide to enzyme catalysis and protein folding. New York: Freeman.

17. Baird, G. S., Zacharias, D. A., \& Tsien, R. Y. (2000). Biochemistry, mutagenesis, and oligomerization of DsRed, a red fluorescent protein from coral. Proceedings of the National Academy of Sciences of the United States of America, 97, 11984-11989.

18. Mizuno, H., Sawano, A., Eli, P., Hama, H., \& Miyawaki, A. (2001). Red fluorescent protein from Discosoma as a fusion tag and a partner for fluorescence resonance energy transfer. Biochemistry, 40, 2502-2510.

19. Shagin, D. A., Barsova, E. V., Yanushevich, Y. G., Fradkov, A. F., Lukyanov, K. A., Labas, Y. A., et al. (2004). GFP-like proteins as ubiquitous Metazoan superfamily: evolution of functional features and structural complexity. Molecular and Biological Evolution, 21, 841-850.

20. Ward, W. W. (1998) Green fluorescent protein: properties, applications, and protocols. In: Chalfie M \& Kain S (Eds). Wiley, New York, pp 45-75.

21. Wiedenmann, J., Elke, C., Spindler, K. D., \& Funke, W. (2000). Cracks in the beta-can: fluorescent proteins from Anemonia sulcata (Anthozoa, Actinaria). Proceedings of the National Academy of Sciences of the United States of America, 97, 14091-14096.

22. Wiedenmann, J., Schenk, A., Rocker, C., Girod, A., Spindler, K. D., \& Nienhaus, G. U. (2002). A far-red fluorescent protein with fast maturation and reduced oligomerization tendency from Entacmaea quadricolor (Anthozoa, Actinaria). Proceedings of the National Academy of Sciences of the United States of America, 99, 11646-11651.

23. Ormo, M., Cubitt, A. B., Kallio, K., Gross, L. A., Tsien, R. Y., \& Remington, S. J. (1996). Crystal structure of the Aequorea victoria green fluorescent protein. Science, 273, 1392-1395.

24. Sniegowski, J. A., Lappe, J. W., Patel, H. N., Huffman, H. A., \& Wachter, R. M. (2005). Base catalysis of chromophore formation in Arg96 and Glu222 variants of green fluorescent protein. The Journal of biological chemistry, 280, 26248-26255.

25. Wood, T. I., Barondeau, D. P., Hitomi, C., Kassmann, C. J., Tainer, J. A., \& Getzoff, E. D. (2005). Defining the role of arginine 96 in green fluorescent protein fluorophore biosynthesis. Biochemistry, 44, 16211-16220.

26. Zagranichny, V. E., Rudenko, N. V., Gorokhovatsky, A. Y., Zakharov, M. V., Balashova, T. A., \& Arseniev, A. S. (2004). Traditional GFP-type cyclization and unexpected fragmentation site in a purple chromoprotein from Anemonia sulcata, asFP595. Biochemistry, 43, 13598-13603.

27. Regan, L. (1993). The design of metal-binding sites in proteins. Annual review of biophysics and biomolecular structure, 22, 257-287.

28. Walter, E. D., Chattopadhyay, M., \& Millhauser, G. L. (2006). The affinity of copper binding to the prion protein octarepeat domain: evidence for negative cooperativity. Biochemistry, 45, 13083-13092.

29. Navarra, G., Leone, M., \& Militello, V. (2007). Thermal aggregation of beta-lactoglobulin in presence of metal ions. Biophysical chemistry, 131, 52-61.

30. Navarra, G., Giacomazza, D., Leone, M., Librizzi, F., Militello, V., \& San Biagio, P. L. (2009). Thermal aggregation and ion-induced cold-gelation of bovine serum albumin. European biophysics journal : EBJ, 38, $437-446$.

31. Vogt, A., D’angelo, C., Oswald, F., Denzel, A., Mazel, C. H., Matz, M. V., et al. (2008). A green fluorescent protein with photoswitchable emission from the deep sea. PloS one, 3, e3766.

32. Sigel, H. (1982) Metal ions in biological systems, properties of copper, vol. 12. Marcel Dekker, New York.

33. Silvia, J., \& Williams, R. (1991). The biological chemistry of the elements: the inorganic chemistry of life. Oxford: Claredon.

34. Fitzgerald, D. J. (1998). Safety guidelines for copper in water. The American journal of clinical nutrition, 67, 1098S-1102S. 